\title{
LA INFLACIÓN MODERADA Y LA INESTABILIDAD DEL PROCESO INFLACIONARIO
}

\author{
Alejandro M. Werner \\ Santiago Bazdresch ${ }^{1}$
}

Junio de 1999

Documento de Investigación No. 9906

Dirección General de Investigación Económica

BANCO DE MÉXICO

${ }^{1}$ Se agradecen los comentarios de Agustín Carstens y Moisés Schwartz Rosenthal en la elaboración de este documento. Las opiniones contenidas en este trabajo corresponden exclusivamente a los autores y no necesariamente representan el punto de vista del Banco de México. 


\title{
LA INFLACIÓN MODERADA Y LA INESTABILIDAD DEL PROCESO INFLACIONARIO
}

\author{
Alejandro M. Werner \\ Santiago Bazdresch \\ Junio de 1999 \\ D ocumento de Investigación No. 9906
}

\begin{abstract}
RESUMEN
La economía mexicana ha experimentado niveles moderados de inflación durante gran parte de su historia reciente. En esta nota se argumenta que no es deseable que un país mantenga estos niveles de inflación por periodos prolongados de tiempo, no sólo porque sus costos a largo plazo son grandes e inevitables, sino porque a medida que se extiende el periodo de inflación moderada, la probabilidad de que la inflación se reduzca en el futuro desciende considerablemente. En este trabajo se realiza un estudio histórico del comportamiento inflacionario de países que han transitado por niveles de inflación moderada por periodos de distinta magnitud. Se comprueba que la probabilidad de mantenerse en esta situación es sustancialmente menor a la de mantenerse en una inflación baja y que la probabilidad de observar un incremento considerable en la tasa de inflación aumenta de manera importante al pasar de un rango de baja inflación a un rango de inflación moderada. Además se encuentra que los procesos desinflacionarios que no han dependido de un ancla cambiaria han tenido resultados más duraderos que aquellos programas basados en un tipo de cambio fijo. Por último, se analizan los casos clásicos de países con inflación moderada.
\end{abstract}




\section{LA IN FLACIÓN MODERADA Y LA INESTABILIDAD DEL PROCESO INFLACIONARIO}

\section{Introducción}

La lucha contra la inflación ha sido uno de los temas más controvertidos y de mayor importancia para nuestro país en las últimas dos décadas. La teoría económica y la evidencia empírica indican que los altos índices inflacionarios son un gran obstáculo para la estabilidad y el crecimiento económico de largo plazo. Además, muchos estudios encuentran que la inflación presenta un componente inercial importante, lo que dificulta su abatimiento una vez que ésta se ha mantenido en niveles elevados por algún tiempo. Por estos motivos, se considera que la reducción de la inflación es uno de los problemas más importantes que enfrenta la economía en la actualidad. Sin embargo, no faltan argumentos en el sentido opuesto, en gran medida debido a la dificultad empírica que han encontrado varios académicos para estimar costos importantes que se deriven directamente de inflaciones "bajas o moderadas". Por estos motivos, se ha argumentado que una vez que se ha alcanzado un nivel inflacionario "moderado", el costo social de reducir la inflación y los costos políticos de emprender un programa anti-inflacionario superan el beneficio que se obtendría de la reducción de la inflación de niveles moderados a bajos. El argumento típico que se presenta en este tipo de discusiones, es que existen ejemplos de países que han sobrellevado altas tasas inflacionarias por más de 15 años consecutivos y que, a primera vista, no han presentado problemas mayores.

En la actualidad existen varios estudios que documentan y cuantifican los costos en términos de crecimiento económico generados por inflaciones elevadas (véase por ejemplo Barro (1995, 1997), Fischer (1993) y De Gregorio (1992, 1993). Además, algunos estudios documentan que estos costos se presentan aún a niveles de baja inflación (véase Sarel (1996) y G hosh y Stevens (1998)). En este estudio se presenta otro aspecto negativo de la inflación moderada, su inestabilidad. Por lo tanto, se argumenta que la reducción de una inflación moderada no sólo tiene efectos positivos 
sobre el crecimiento económico, sino que también contribuye sustancialmente a reducir la probabilidad de transitar a niveles de inflación elevada en el futuro

En este trabajo se estudia la relación entre los niveles de inflación y su estabilidad. Para estos efectos se analiza la experiencia inflacionaria internacional para el periodo 19481996 poniendo atención especial en las transiciones entre niveles de inflación bajos, moderados y altos. Este estudio confirma que los niveles moderados de inflación son sustancialmente mas inestables que los niveles de inflación baja y que, si bien es cierto que si una economía convive un tiempo prolongado con inflación moderada, ésta se vuelve más estable, este fenómeno ocurre a costa de disminuir la probabilidad de que la inflación descienda a niveles "bajos" mientras que la probabilidad de tránsito al rango de inflación "alta" permanece constante y en niveles considerables. Este resultado se ve acentuado si la economía que mantiene un nivel de inflación moderado, llegó a este rango proveniente de niveles de inflación alta. Finalmente, se estudian algunos casos de países que transitaron por un régimen de inflación moderada y lograron reducirla y los casos de aquellos países que no fueron exitosos en la reducción de la tasa de inflación. De este estudio de casos, se desprende que existen muy pocos episodios de inflación moderada de larga duración. Además, se observa claramente cómo estos niveles moderados de inflación han aumentado de manera considerable la vulnerabilidad de las economías ante perturbaciones de origen externo e interno y como estas economías al enfrentarse a estas perturbaciones han transitado rápidamente a niveles elevados de inflación.

\section{La Estabilidad del Proceso Inflacionario: Experiencia Internacional}

\section{II.1 Metodología y descripción de los datos}

En primer lugar se obtuvieron los datos de inflación para una muestra amplia de países. Se utilizó el Indice de Precios al Consumidor reportado por el FMI durante el periodo 1948 -1996, para 139 países. Muchos países no cuentan con información que llegue hasta 1996, además de que para algunos países los datos se ven interrumpidos por crisis políticas, o tienen muy poco tiempo de existir como entidades con unidad monetaria definida. Por lo tanto, se decidió desechar aquellos países que tienen menos de 10 registros anuales de precios al consumidor en este intervalo de tiempo. 
Después de realizar la depuración mencionada, se cuenta con información para 128 países. Para cada país, y para cada año con información disponible, se determinó si la inflación fue baja, moderada 0 alta. Se consideró como inflación alta aquélla superior al $20 \%$, moderada la que se encuentra entre 10 y $20 \%$ y baja la que es menor a $10 \%$. Se incluyen los periodos de deflación dentro del rango de inflación baja, pues existen muy pocos de éstos episodios que justificar su inclusión por separado. Esta clasificación es un poco arbitraria, pero en alguna medida coincide con las utilizadas en otros trabajos sobre el comportamiento del crecimiento de los precios. ${ }^{2}$ Posteriormente, se realiza una clasificación más fina de los rangos inflacionarios.

Una vez hecha esta clasificación se determinó para cada país, cuáles son los episodios de inflación baja, moderada o alta de uno hasta cinco años consecutivos de duración. Por ejemplo, un episodio de tres años en inflación baja es aquél en el que un país tuvo una inflación moderada o alta un año e inmediatamente después se observan tres años consecutivos o más de inflación baja. Es claro que hay más casos de uno o dos años con inflación similar que episodios de cinco años consecutivos. En particular, no es fácil que un país se mantenga mucho tiempo con una inflación que oscile entre $10 \mathrm{y}$ $20 \%$.

Una vez identificados los episodios, se observó la situación inflacionaria del país inmediatamente después de haber concluido éstos y la situación promedio de los 5 años posteriores. Los diferentes horizontes permiten distinguir si alguno de los resultados está determinado por movimientos transitorios de la inflación entre los diferentes rangos. Por ejemplo, si un país tuvo inflación moderada por cinco años consecutivos y logra reducirla, existe una alta probabilidad de que regrese al rango moderado algunos años después.

La información obtenida se resume en valores probabilísticos. Para hacerlo basta contar y dividir. Se cuenta el número de episodios de cada tipo (inflación alta, moderada o baja, de uno a cinco años de duración), y cuántos de éstos tuvieron inflación alta, moderada o baja, un año después o como promedio de los próximos 5 años. Se dividen los casos entre el total y obtenemos el resultado. Esto quiere decir

\footnotetext{
${ }^{2}$ Por ejemplo, Dornbusch y Fischer (1991).
} 
que para cada tipo de episodio inflacionario se conoce qué proporción de los países en esta situación terminaron con inflación alta, moderada 0 baja. Estos valores representan la probabilidad de que un país pase de un rango de inflación a otro, por lo que estas proporciones se denominarán las "probabilidades de transición" entre un estado inflacionario y otro.

Finalmente, esta información se expresa de una manera que facilita su entendimiento e interpretación. Esto se realiza tomando en cuenta cuáles son los tres elementos variables del estudio: el número de períodos consecutivos que un país tuvo inflación en el mismo rango, cuál es este rango, y el tiempo después del episodio inflacionario que tomamos para observar el resultado, un año o el promedio de cinco años. Para presentar los resultados se fija cada una de estas variables y se observa la relación experimentada entre las otras dos. Los resultados se presentan con varias "matrices de transición" y algunas gráficas.

A continuación se ejemplifica el procedimiento de clasificación de los episodios utilizando la experiencia de Irlanda y Turquía durante el periodo 1970-1981. El primer cuadro indica la inflación anual en cada uno de estos países.

\section{Cuadro 1: Inflación Anual en Irlanda y Turquía}

\begin{tabular}{|c|l|l|l|l|c|c|c|c|c|c|c|c|}
\hline Año & 1970 & 1971 & 1972 & 1973 & 1974 & 1975 & 1976 & 1977 & 1978 & 1979 & 1980 & 1981 \\
\hline Irlanda & 8.21 & 8.96 & 8.61 & 11.41 & 16.98 & 20.88 & 17.99 & 13.64 & 7.63 & 13.24 & 18.22 & 20.34 \\
\hline Turquía & 6.93 & 15.74 & 11.67 & 15.44 & 15.82 & 19.20 & 17.36 & 27.08 & 45.28 & 58.69 & 110.1 & 36.57 \\
\hline
\end{tabular}

Clasificamos cada una de estas inflaciones anuales como baja (B), moderada (M) 0 alta (A):

Cuadro 2: Clasificación de los Episodios Inflacionarios

\begin{tabular}{|c|c|c|c|c|c|c|c|c|c|c|c|c|}
\hline Año & 1970 & 1971 & 1972 & 1973 & 1974 & 1975 & 1976 & 1977 & 1978 & 1979 & 1980 & 1981 \\
\hline Irlanda & $\mathrm{B}$ & $\mathrm{B}$ & $\mathrm{B}$ & $\mathrm{M}$ & $\mathrm{M}$ & $\mathrm{A}$ & $\mathrm{M}$ & $\mathrm{M}$ & $\mathrm{B}$ & $\mathrm{M}$ & $\mathrm{M}$ & $\mathrm{A}$ \\
\hline Turquía & $\mathrm{B}$ & $\mathrm{M}$ & $\mathrm{M}$ & $\mathrm{M}$ & $\mathrm{M}$ & $\mathrm{M}$ & $\mathrm{M}$ & $\mathrm{A}$ & $\mathrm{A}$ & $\mathrm{A}$ & $\mathrm{A}$ & $\mathrm{A}$ \\
\hline
\end{tabular}


En primer lugar, se identifican los episodios de inflación baja, moderada 0 alta. En este ejemplo se estudian los episodios de dos años o más de duración. Por lo tanto, tenemos a Irlanda, empezando en 1970 con inflación baja; y en 1973, 1976 y 1979 con inflación moderada, y Turquía, empezando en 1971 con inflación moderada, y en 1977 con inflación alta.

Se analiza el resultado de cada uno de estos episodios un año después del segundo año, y se observa que Irlanda en 1972 y 1978 tuvo inflación baja, y en 1975 y 1981 inflación alta, mientras que Turquía en 1973 tuvo inflación moderada y en 1979 inflación alta.

Estos resultados se conjuntan de la siguiente manera. Todos los episodios de inflación baja (Irlanda, 1970) tuvieron en el tercer año inflación baja, uno de cuatro episodios de inflación moderada experimentó inflación moderada (Turquía 1971), dos inflación alta, (Irlanda 1976 y 1979) y uno de ellos inflación baja (Irlanda 1976). Finalmente, todos los episodios de inflación alta terminaron en inflación alta (Turquía 1977). A partir de estos resultados se construye la siguiente matriz:

\section{Cuadro 3: Transiciones Inflacionarias en Irlanda y Turquía}

\begin{tabular}{|c|c|c|c|c|}
\hline Rango & B & M & A & Total de casos \\
\hline B & 1 & 0 & 0 & 1 \\
\hline M & 1 & 1 & 2 & 4 \\
\hline A & 0 & 0 & 1 & 1 \\
\hline
\end{tabular}

La cual puede expresarse en términos de probabilidades la siguiente manera:

\section{Cuadro 4: Matriz de Transición para Irlanda y Turquía}

\begin{tabular}{|c|c|c|c|}
\hline Rango & B & M & A \\
\hline B & $\mathbf{1 0 0} \%$ & $\mathbf{0} \%$ & $\mathbf{0} \%$ \\
\hline M & $\mathbf{2 5 \%}$ & $\mathbf{2 5} \%$ & $\mathbf{5 0} \%$ \\
\hline A & $\mathbf{0} \%$ & $\mathbf{0} \%$ & $\mathbf{1 0 0} \%$ \\
\hline
\end{tabular}


Los valores de esta matriz son las probabilidades depaso o detransián entre un estado y otro que se obtuvieron a partir de los datos disponibles. Esta es sólo una de tantas posibilidades, pues se consideraron únicamente los episodios de dos años o más de duración y se observó en dónde se encontraba la inflación al tercer año de comenzado el episodio.

Por ejemplo, el primer renglón de esta matriz nos indica las probabilidades de que una economía que haya estado por dos años en el rango de inflación baja pase al año siguiente al rango de inflación baja, moderada o alta, respectivamente. Dado que estas son las únicas tres alternativas, la suma de estas probabilidades debe ser igual a 1.

\section{II.2 Resultados generales}

Los resultados básicos del análisis son las siguientes matrices de transición, en las que se reporta la probabilidad con la que un país pasa de un rango inflacionario a otro. A continuación, se presentan las probabilidades de transición a diferentes rangos inflacionarios en el año siguiente, para el caso en el que un país ha estado en el mismo rango inflacionario por dos años.

\section{Cuadro 5: Matriz de Transición para episodios de dos años, vistos un año después}

\begin{tabular}{|l|c|c|c|}
\hline \multicolumn{1}{|c|}{ Rango } & Baja & Moderada & Alta \\
\hline Baja & $\mathbf{7 4 . 1 \%}$ & $\mathbf{2 3 . 3 \%}$ & $\mathbf{2 . 6 \%}$ \\
\hline Moderada & $\mathbf{3 5 . 2} \%$ & $\mathbf{4 6 . 0} \%$ & $\mathbf{1 8 . 8} \%$ \\
\hline Alta & $\mathbf{1 5 . 3} \%$ & $\mathbf{2 2 . 4 \%}$ & $\mathbf{6 2 . 2} \%$ \\
\hline
\end{tabular}

De las probabilidades de continuar en el mismo rango inflacionario, (las cifras en la diagonal) la de la inflación moderada es la más baja, con un valor de $46.0 \%$ comparado con $74.1 \%$ y $62.2 \%$ para inflaciones bajas y altas, respectivamente. Por lo tanto, para un país que lleva dos años en inflación moderada existe prácticamente la misma 
probabilidad de permanecer en ese rango que de pasar a algún otro. Al comparar la probabilidad de llegar a una inflación alta a partir de inflación baja y moderada se observa que la primera es de $2.6 \%$ mientras que la segunda se encuentra en $18.8 \%$. Desde esta perspectiva un país con inflación moderada es mucho más propenso que un país con inflación baja a transitar hacia una inflación elevada, al menos en el corto plazo. A continuación, se realizó el mismo ejercicio pero se amplía el horizonte, esto es, se presentan las probabilidades de transición en términos del promedio inflacionario de los próximos cinco años para aquellos episodios en los cuales la inflación ha permanecido en el mismo rango por 2 años.

\section{Cuadro 6: Matriz de Transición para el promedio de cinco años}

\begin{tabular}{|l|c|c|c|}
\hline Rango & Baja & Moderada & Alta \\
\hline Baja & $\mathbf{6 9 . 5 \%}$ & $\mathbf{2 3 . 6} \%$ & $\mathbf{6 . 9 \%}$ \\
\hline Moderada & $\mathbf{3 9 . 7} \%$ & $\mathbf{4 7 . 4 \%}$ & $\mathbf{1 2 . 9} \%$ \\
\hline Alta & $\mathbf{9 . 1} \%$ & $\mathbf{3 5 . 2} \%$ & $\mathbf{5 5 . 7} \%$ \\
\hline
\end{tabular}

En cuanto a las probabilidades de permanecer en el mismo rango se obtienen resultados muy similares con el promedio que con el resultado de más corto plazo. La probabilidad de tener un promedio de inflación moderada partiendo de una inflación en el mismo rango es menor al 50\%. Por otra parte, la probabilidad de pasar a una inflación alta a partir de inflación moderada disminuye al considerar el promedio de los próximos 5 años, y la de pasar a inflación baja aumenta. Finalmente, se detectan cambios significativos en las probabilidades de pasar a inflación baja 0 moderada a partir de inflación alta, en particular es más difícil mantener un promedio de inflación baja que tener un año de inflación baja.

A continuación se extiende este análisis definiendo los intervalos inflacionarios de una manera más precisa. Con este propósito, se determinan seis rangos inflacionarios, cinco de diez puntos porcentuales a partir de $0 \%$ y un último para los valores mayores a 50\%. Al realizar este ejercicio, se esclarecen varios fenómenos. Sin embargo, al aumentar el número de rangos inflacionarios, se presenta una desventaja ya que el número disponible de casos en cada rango disminuye. 


\section{Cuadro 7: Matriz de Transición para Episodios de dos años, un año después.}

\begin{tabular}{|c|c|c|c|c|c|c|}
\hline \& & $\frac{\delta^{0}}{\sigma^{\prime}}$ & $\begin{array}{l}\overbrace{0}^{\circ} \\
\text { o }\end{array}$ & $\begin{array}{l}\text { ठे } \\
\text { 乃े } \\
\text { ô }\end{array}$ & $\begin{array}{l}\text { ठి } \\
\text { ఫे } \\
\text { के }\end{array}$ & $\begin{array}{l}\text { ठे } \\
\text { ధ } \\
\text { of }\end{array}$ & 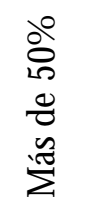 \\
\hline $0-10 \%$ & $73.1 \%$ & $24.1 \%$ & $2.1 \%$ & $0.7 \%$ & $0.0 \%$ & $0.0 \%$ \\
\hline $10-20 \%$ & $33.8 \%$ & $46.8 \%$ & $14.0 \%$ & $3.6 \%$ & $0.5 \%$ & $1.4 \%$ \\
\hline $20-30 \%$ & $10.3 \%$ & $25.9 \%$ & $43.1 \%$ & $12.1 \%$ & $1.7 \%$ & $6.9 \%$ \\
\hline $30-40 \%$ & $10.0 \%$ & $15.0 \%$ & $20.0 \%$ & $25.0 \%$ & $15.0 \%$ & $15.0 \%$ \\
\hline $40-50 \%$ & $0.0 \%$ & $0.0 \%$ & $18.2 \%$ & $27.3 \%$ & $9.1 \%$ & $45.5 \%$ \\
\hline $\begin{array}{|ll|}\text { más } & \text { de } \\
50 \% & \\
\end{array}$ & $3.0 \%$ & $0.0 \%$ & $9.1 \%$ & $6.1 \%$ & $6.1 \%$ & $75.8 \%$ \\
\hline
\end{tabular}

\begin{tabular}{|c|c|c|}
\hline  &  & 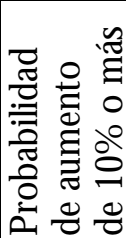 \\
\hline - & $26.9 \%$ & $2.8 \%$ \\
\hline $33.8 \%$ & $19.4 \% \%$ & $5.4 \%$ \\
\hline $36.2 \%$ & $20.7 \%$ & $8.6 \%$ \\
\hline $45.0 \%$ & $30.0 \%$ & $15.0 \%$ \\
\hline $45.5 \%$ & $45.5 \%$ & $9.1 \%$ \\
\hline $24.2 \%$ & - & - \\
\hline
\end{tabular}

Lo primero que se observa es que las probabilidades de que la inflación se mantenga dentro del mismo rango disminuyen conforme pasamos a rangos de mayor inflación. Este resultado reafirma la intuición y experiencia de series de tiempo que indican que en la medida que la inflación aumenta también se vuelve más volátil e incierta. Además, se calculó la probabilidad de pasar a un rango inflacionario mayor en cada uno de los casos. Se observa cómo esta probabilidad crece al aumentar la inflación de donde se parte, pasando de $19.4 \%$ para el rango de entre 10 y 20\% hasta casi 50\% para el último rango de diez puntos porcentuales. La probabilidad de que se observe un aumento en la tasa de inflación para el rango de 0 a $10 \%$ es mayor que para los 2 rangos siguientes, pero estos aumentos son pequeños ya que la probabilidad de observar un aumento de mas de 10 puntos porcentuales sigue siendo muy baja. Se obtiene un resultado similar para la probabilidad de observar un aumento de 10 puntos porcentuales de inflación en el periodo siguiente, ya que la probabilidad de que esto ocurra casi se duplica al pasar del rango de 0-10\% al de 10-20\% y alcanza un valor de $15 \%$ en el rango de 30 a 40\% y disminuye al pasar al rango de 40 a 50\%, lo que puede indicar que a estos niveles se generan presiones significativas para implementar programas desinflacionarios. 


\section{Cuadro 8: Matriz de Transición para Episodios de dos años}

(promedio de cinco años)

\begin{tabular}{|c|c|c|c|c|c|c|c|c|c|}
\hline $\begin{array}{l}\text { क्ष్ } \\
\text { क्ल } \\
\end{array}$ & $\begin{array}{l}80 \\
\text { రे }\end{array}$ & ठ్రి & $\begin{array}{l}\text { ठे } \\
\text { రे }\end{array}$ & $\begin{array}{l}\text { ఫे } \\
\text { ஓे }\end{array}$ & $\begin{array}{l}\text { ठे? } \\
\text { } \\
\text { ᄋे }\end{array}$ & $\begin{array}{l}80 \\
80 \\
8 \\
8 \\
0 \\
0\end{array}$ & 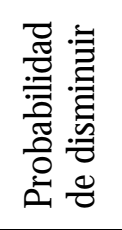 &  & 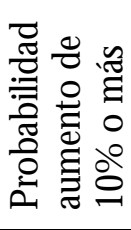 \\
\hline $0-10 \%$ & $68.3 \%$ & $25.4 \%$ & $3.7 \%$ & $2.2 \%$ & $0.0 \%$ & $\mathbf{0 . 4 \%}$ & - & $31.7 \%$ & $6.3 \%$ \\
\hline $10-20 \%$ & $38.9 \%$ & $47.6 \%$ & $7.7 \%$ & $2.4 \%$ & $1.0 \%$ & $2.4 \%$ & $38.9 \%$ & $13.5 \%$ & $5.8 \%$ \\
\hline $20-30 \%$ & $8.7 \%$ & $41.3 \%$ & $26.1 \%$ & $8.7 \%$ & $2.2 \%$ & $13.0 \%$ & $50.0 \%$ & $23.9 \%$ & $15.2 \%$ \\
\hline $30-40 \%$ & $5.6 \%$ & $16.7 \%$ & $11.1 \%$ & $22.2 \%$ & $11.1 \%$ & $33.3 \%$ & $33.3 \%$ & $44.4 \%$ & $33.3 \%$ \\
\hline $40-50 \%$ & $0.0 \%$ & $11.1 \%$ & $22.2 \%$ & $22.2 \%$ & $22.2 \%$ & $22.2 \%$ & $55.6 \%$ & $22.2 \%$ & $22.2 \%$ \\
\hline $\begin{array}{l}\text { más de } \\
50 \%\end{array}$ & $\mathbf{0 . 0} \%$ & $3.3 \%$ & $3.3 \%$ & $10.0 \%$ & $13.3 \%$ & $70.0 \%$ & $30.0 \%$ & - & - \\
\hline
\end{tabular}

Esta segunda matriz de seis rangos inflacionarios resume lo sucedido en promedio en los 5 años siguientes al segundo año del episodio inflacionario. La estructura general es muy similar a la matriz anterior, pero vale la pena destacar las diferencias. Para todos los rangos inflacionarios, la probabilidad de que la inflación disminuya es mayor si se observa el promedio de los siguientes cinco años que si se observa el resultado del año siguiente. En varios casos, la probabilidad de aumentar de rango inflacionario es mayor al observar el promedio. Para la inflación entre 40 y 50\%, tenemos que cuando se trata del promedio, la probabilidad de aumentar de rango inflacionario disminuye fuertemente, de $50 \%$ a $22 \%$ y la de pasar a una inflación menor aumenta de $41 \%$ a $55.6 \%$. Esto puede deberse a que una vez que se alcanzan estos niveles, los gobiernos han decidido estabilizar ya que la presión política para tal efecto aumenta de manera considerable. 


\section{II.3 La inflación moderada}

Esta sección se concentra en los resultados obtenidos para la inflación moderada, es decir el intervalo entre 10 y 20\% de inflación. En particular, se estudia cómo cambian las probabilidades de transición a inflación baja 0 alta cuando un país ha experimentado una inflación moderada por un periodo de tiempo determinado.

La discusión en torno a la inflación moderada sugiere que después de que un país ha estado algunos años dentro de este rango, la economía parece ajustarse a estos niveles y no hay ninguna razón aparente para hacer un esfuerzo por reducir la tasa de crecimiento de los precios. En principio, se podría pensar que cuantos más años un país permanece en inflación moderada, la probabilidad de que ésta se mantenga en este rango aumenta, es decir, este rango se vuelve más estable. En la siguiente gráfica se estudia si los resultados confirman esta hipótesis. Para este fin, en la gráfica 1, se presenta la probabilidad de transición a inflaciones bajas, moderadas y altas un año después de que una economía ha estado de 1 a 5 años en el rango de inflación moderada.

Gráfica 1

Probabilidad de transición un año después de un episodio en inflación moderada

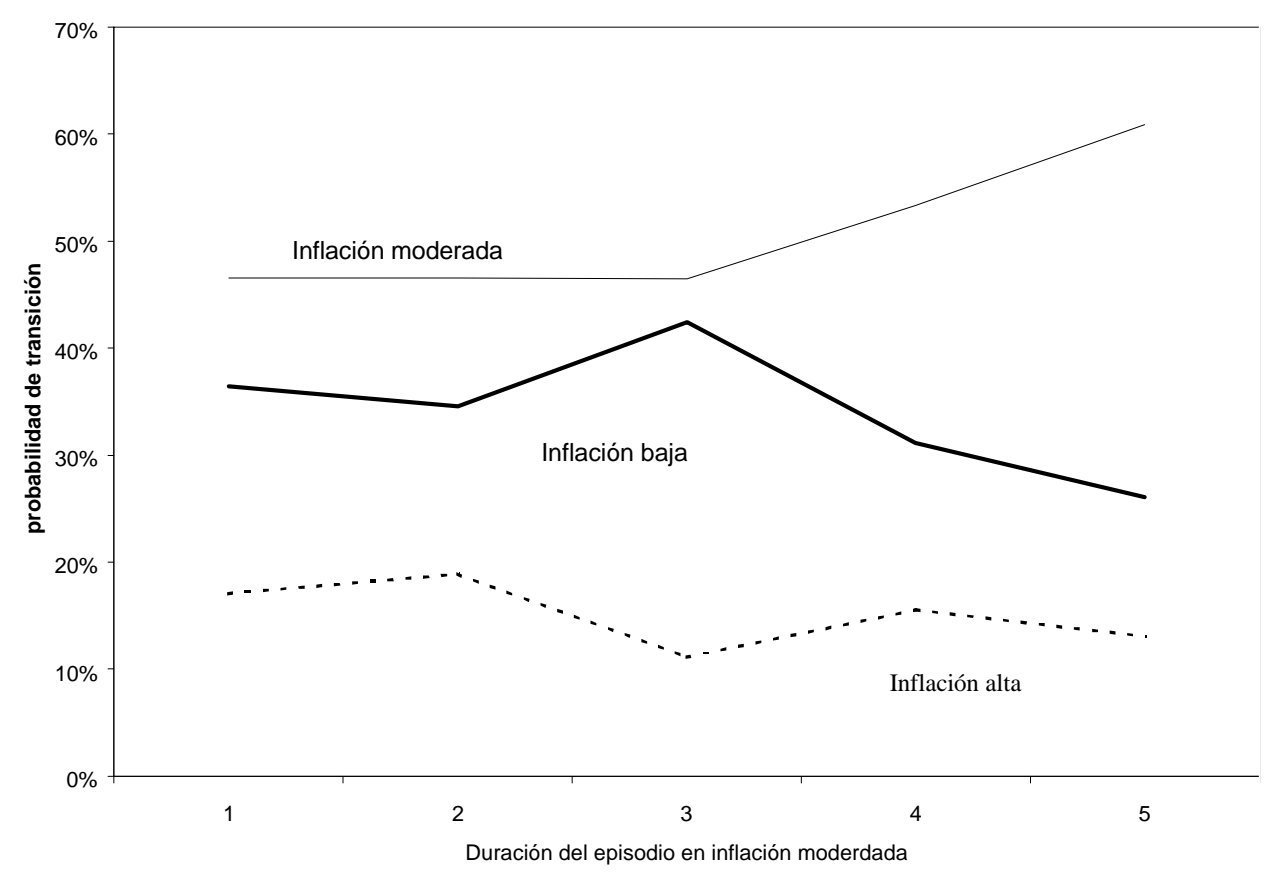


A partir de esta gráfica se observa cómo, si un país pasa más tiempo en el rango de inflación moderada, la probabilidad de que se encuentre en este rango el año siguiente aumenta y disminuye la probabilidad de que se encuentre en el rango de inflación baja. Con esta información podríamos pensar que la inflación moderada se vuelve progresivamente más estable. Sin embargo, al observar lo que sucede con la probabilidad de transición a inflación alta, vemos que ésta no cambia significativamente. Por lo tanto, la inflación moderada se vuelve progresivamente más estable debido a que disminuye la probabilidad de que la inflación se reduzca en el futuro, ya que la probabilidad de transición al rango de inflación elevada se mantiene más o menos constante en niveles de $15 \%$ a partir del tercer año. Al parecer, lo que sucede es que después e algunos años en los que la inflación se encuentra en el rango moderado, la economía desarrolla mecanismos de defensa ante estos niveles inflacionarios que dificultan el descenso de la inflación en el futuro. Esto no quiere decir que la inflación se ha estabilizado a estos niveles, pues la probabilidad de pasar a un rango inflacionario mayor sigue siendo relativamente elevada. D e hecho, se observa que aún después de que una economía ha estado cinco años en el rango de inflación moderada existe una probabilidad de 15\% de encontrarse en el rango de inflación alta el año siguiente.

Tomando en cuenta estos resultados, resulta clara la importancia de reducir la inflación antes de que la economía se adapte a estos niveles y la capacidad de reducirla en el futuro disminuya.

En muchos casos, se han observado reducciones transitorias de los niveles de inflación moderados a bajos por lo que los resultados anteriores pueden verse influidos por estos fenómenos. Para corregir este efecto, en la gráfica 2 se estudia cómo cambia la gráfica anterior si se toman los resultados promedio cinco años después de los episodios de inflación moderada de 1 a 5 años de duración. 
Gráfica 2

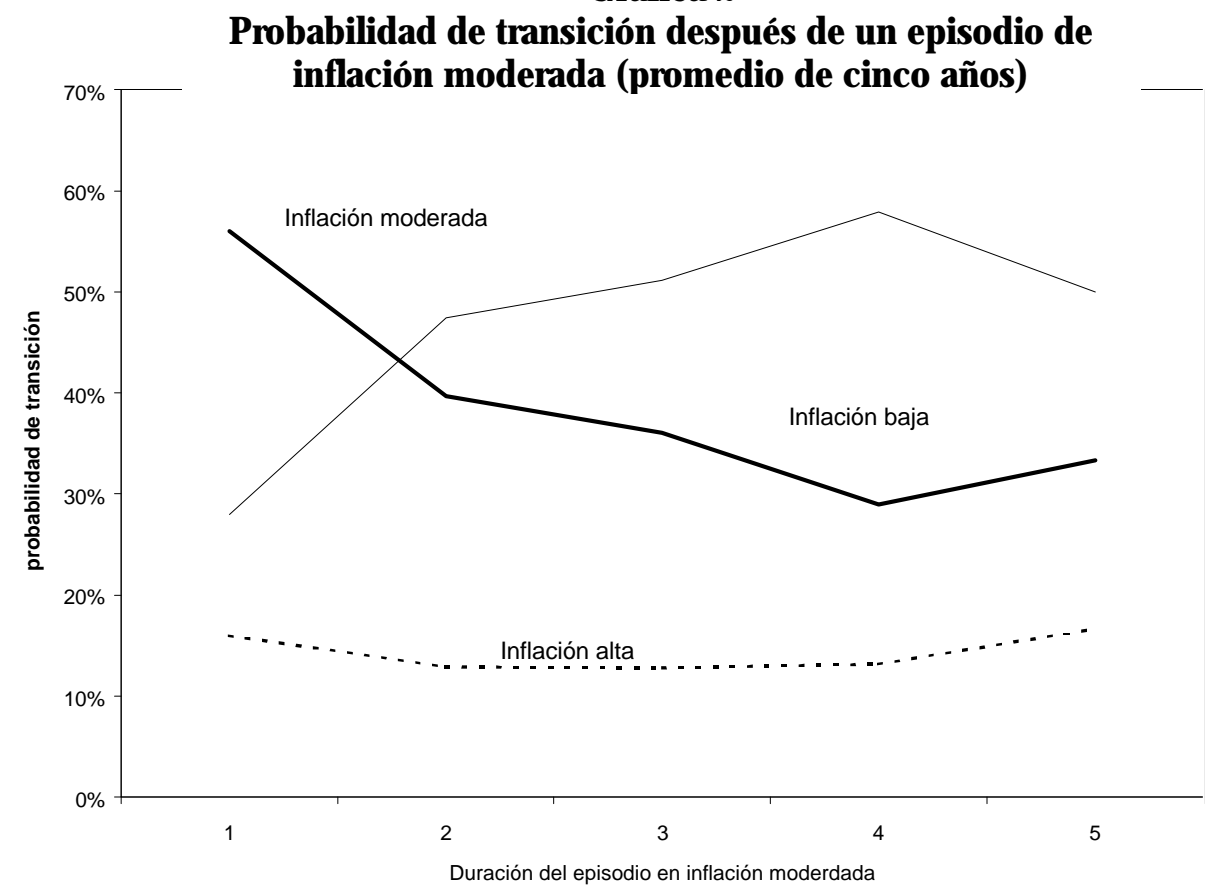

En esta gráfica se observa cómo cuando una economía se encuentra más años consecutivos en el rango de inflación moderada, las diferencias en las probabilidades de transición se acentúan. La probabilidad de tener en promedio inflación baja después de haber pasado un año en inflación moderada es de 56.0\% mientras que la probabilidad de estar de nuevo en el rango moderado es de sólo 28.0\%. Finalmente, si consideramos lo sucedido con los episodios de cinco años de duración, la probabilidad de que una economía experimente su inflación promedio moderada para los próximos cinco años es de 50.0\% comparada con 33.3\% para el caso de inflación baja y $16.7 \%$ para la alta. Por lo tanto, de este ejercicio se concluye que una vez que han transcurrido algunos años en los que se experimenta una inflación moderada, la probabilidad de quedarnos en este rango en el futuro, es decir su estabilidad, aumenta a costa de que se dé una disminución en la probabilidad de que la inflación disminuya.

Por último, para comparar con los resultados anteriores, a continuación se presentan los resultados equivalentes de aquellas economías que han experimentado inflaciones bajas por un periodo de tiempo determinado. Aún cuando la inflación baja es un rango extremo y no intermedio como la inflación moderada, las magnitudes representadas en estas gráficas son muy significativas. En primer lugar, en la gráfica 3 se presentan las probabilidades de transición a los diferentes rangos inflacionarios en el año siguiente y en la gráfica 4 se presentan los resultados con respecto al promedio 
para los próximos 5 años para el caso en el que una economía ha estado entre 1 y 5 años en inflación baja.

Las diferencias con las cifras presentadas para el caso de la inflación moderada son claras, la probabilidad de que una economía permanezca en el rango de inflación baja es alrededor de $75 \%$ en su punto más bajo, y alcanza valores superiores al $90 \%$, después de 5 años. Es decir, si en un país se observó una inflación baja por 4 ó 5 años, existe una probabilidad mayor al $90 \%$ de que se encuentre en este rango el año siguiente, mientras que la probabilidad de llegar a una inflación alta es menor a 5\%.

A continuación, se presentan los resultados relativos a los episodios de inflación superior al 20\% (inflación alta). Estos resultados sirven para comparar con la información para inflación moderada y baja.

Probabilidad de transición un año después de un episodio en inflación alta

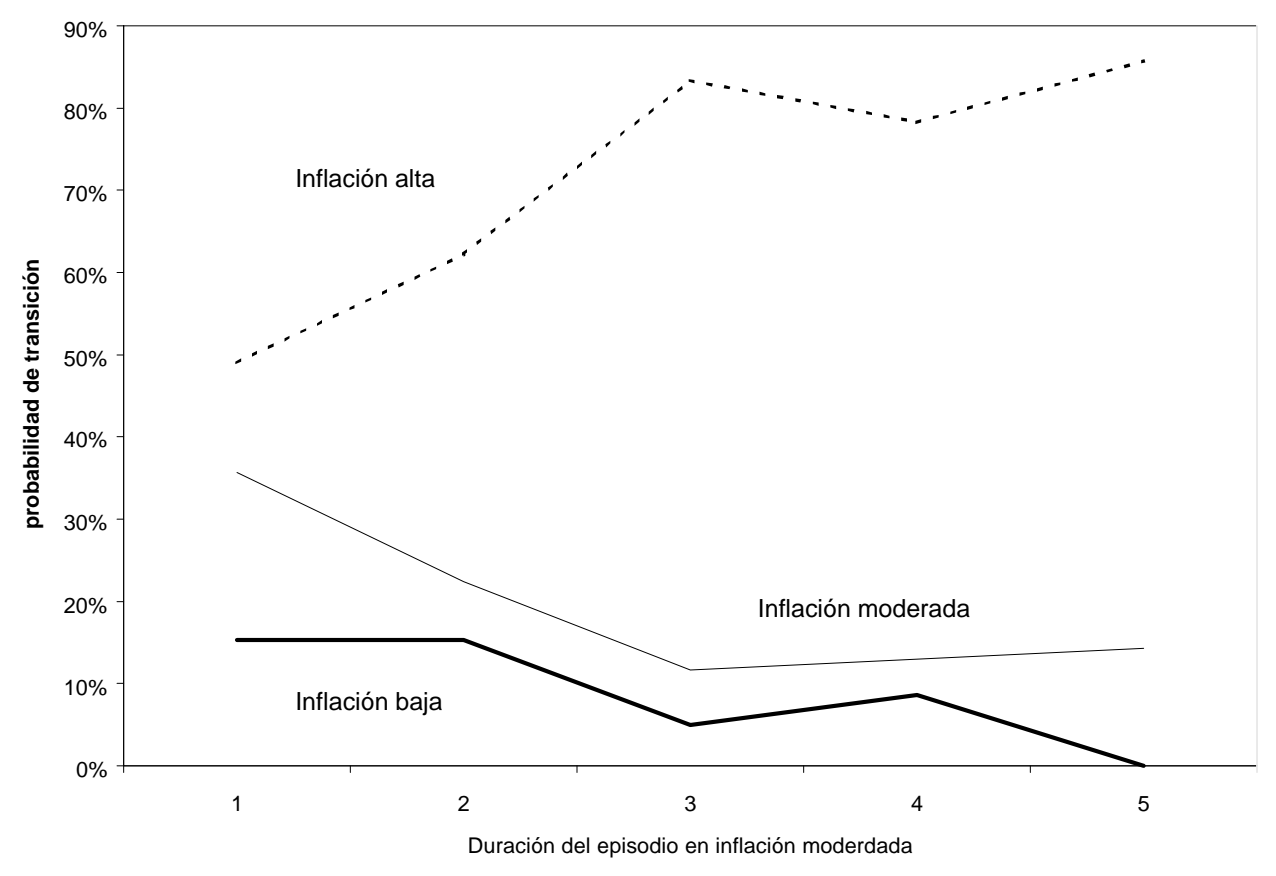


Se puede ver que la inflación alta es más estable que la inflación moderada y depende en mayor medida del número de periodos transcurridos en el rango de inflación alta. También es notable que la probabilidad de pasar a inflación baja es menor a 20\% y es prácticamente cero para episodios de cinco años de duración. Por último presentamos también la gráfica de los promedios de cinco años.

\section{Gráfica 6}

Probabilidad de transición después de un episodio de inflación alta (promedio de cinco años)

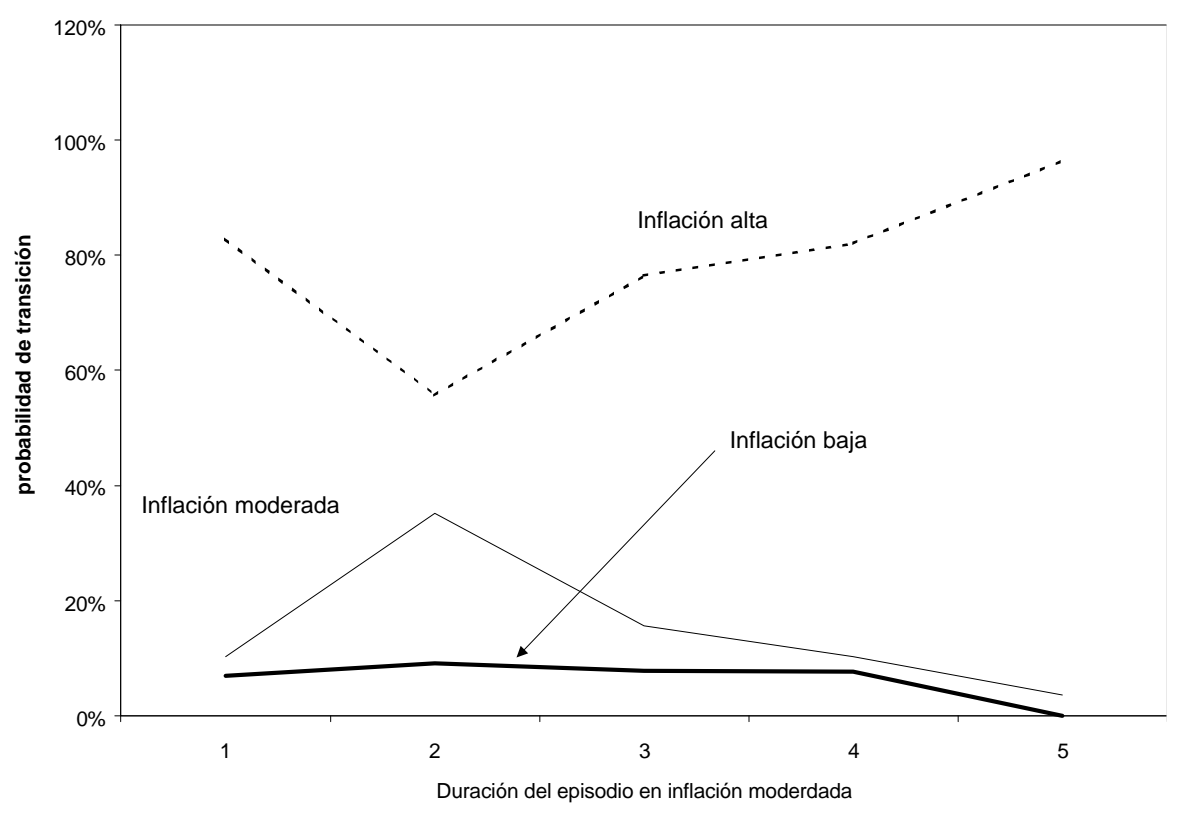

Nuevamente observamos que existe una dependencia importante entre las probabilidades de transición y el número de periodos que la economía ha tenido una inflación alta. En el promedio es mucho más difícil pasar de inflación alta a inflación baja, al parecer es más fácil reducir la tasa inflacionaria por un solo periodo que mantenerla en este nivel por cinco años consecutivos. La probabilidad de pasar a inflación baja en este caso es menor a 10\% en todos los casos y de nuevo es prácticamente cero para los episodios de cinco años de duración.

Hasta este punto se obtuvieron resultados muy ilustrativos a partir de considerar la duración de un episodio de inflación moderada y lo sucedido después de éste. Sin embargo, la historia inflacionaria previa a cada uno de estos episodios es una variable 
que no se ha tomado en cuenta y que puede contribuir a explicar diferencias importantes. Por ejemplo, se puede pensar que sólo los países que vienen de inflación alta tienden a regresar a este rango después de muchos periodos en inflación moderada, o que son éstos los que tienden a permanecer en el rango moderado. Para analizar esta posibilidad, se tomó el rango inflacionario de la economía antes de cada uno de los episodios de inflación moderada y los episodios se separaron entre aquellos que provienen de inflación alta y aquellos que provienen de inflación baja. Aunque el pasado inflacionario de un país no se puede resumir con un solo valor, el rango en que se encuentra la inflación en año antes de iniciar un episodio de inflación moderada conlleva suficiente información de la historia de esta variable como para utilizarlo como variable de selección.

Al realizar esta separación, se encuentra que existe una diferencia importante en las probabilidades de transición dependiendo si antes del episodio de inflación moderada, esta economía tuvo inflación alta o baja. A continuación, se comparan las probabilidades de transición de un episodio en inflación moderada de entre 1 y 5 años de duración, un año después, dividiendo la muestra de países en estos dos grupos.

Gráfica 7

Probabilidad de transición, un año después para episodios de inflación moderada que provienen de inflación baja

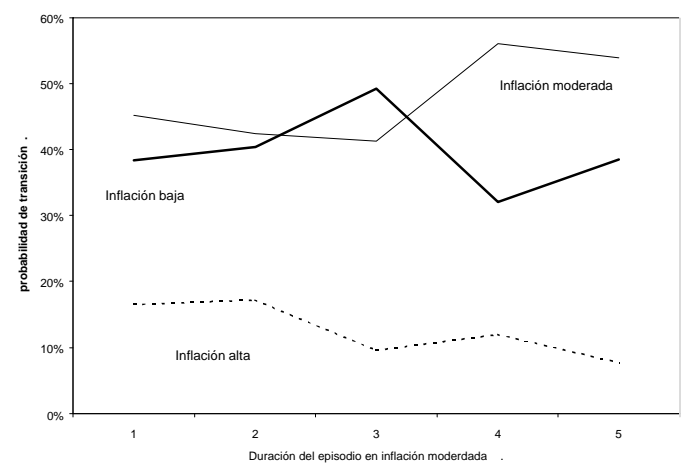

Gráfica 8

Probabilidad de transición, un año después para episodios de inflación moderada que provienen de inflación alta

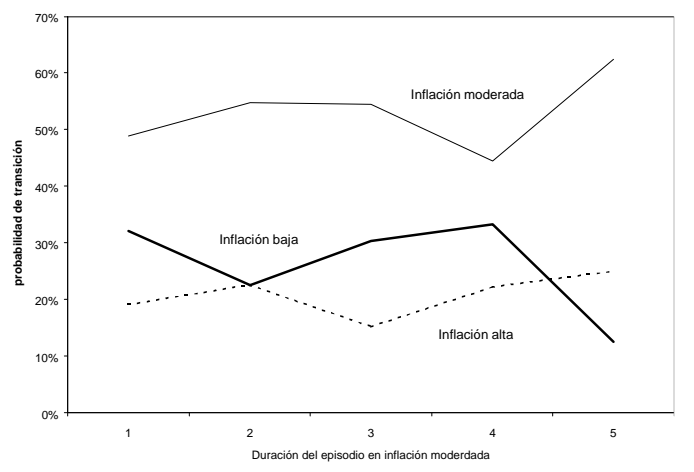

En estas gráficas se presentan las diferencias entre los episodios que provienen de inflación baja y los que provienen de inflación alta. En los primeros hay una probabilidad muy pequeña de llegar a inflación alta (menor a 10\%), y probabilidades similares (de entre 40 y 55\%) de ir al rango moderado y bajo, mientras que para el 
segundo grupo la probabilidad de llegar a inflación alta es mucho mayor (superior a $20 \%$ ), y esta probabilidad es similar a la de llegar a inflación baja. Otra diferencia significativa es que para aquellos casos que provienen de inflación baja, la probabilidad de llegar a inflación alta disminuye al aumentar la duración del episodio en el rango moderado, mientras que para los casos que provienen de la inflación alta esta probabilidad aumenta con el tiempo.

A pesar de las diferencias, ambas gráficas muestran cómo el comportamiento de las probabilidades de llegar a inflación baja y moderada está estrechamente relacionado ya que siempre que aumenta la probabilidad de mantenerse en inflación moderada disminuye la probabilidad de llegar al rango bajo.

Nuevamente resulta importante considerar la trascendencia en el tiempo de los efectos estudiados, por lo cual mostramos las gráficas correspondientes al promedio de los próximos 5 años después de 1 a 5 años en inflación moderada.

Gráfica 9

Probabilidad de transición para episodios de inflación moderada que proviene de inflación baia.

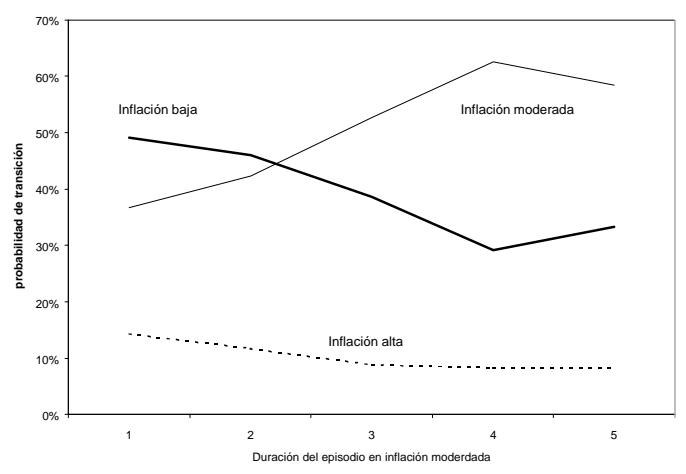

Gráfica 10

Probabilidad de transición para episodios de inflación moderada que proviene de inflación alta.

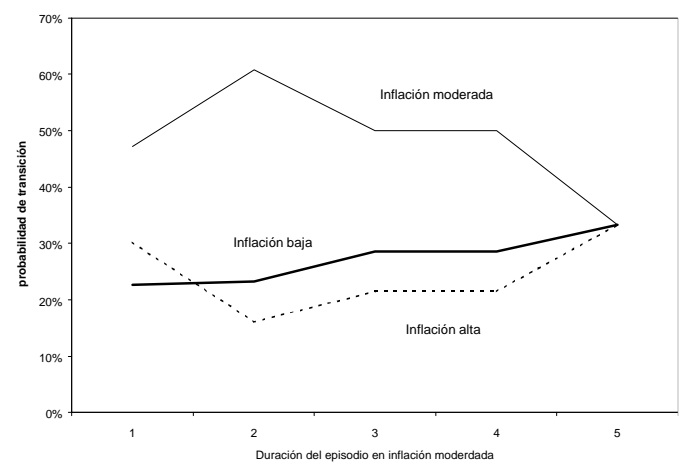

En estas gráficas la evolución en el tiempo es más importante. Nuevamente se observa un comportamiento opuesto entre la probabilidad de llegar a inflación moderada y la de llegar a inflación baja, aunque en este caso es menos pronunciado. También observamos que la probabilidad de llegar a inflación alta es sustancialmente mayor para aquellos episodios que provienen de inflación alta (30\%), que para los episodios que provienen de inflación baja (10\%). Finalmente, se detectó una clara señal de inestabilidad de los episodios que provienen de inflación alta, pues al aumentar la cantidad de periodos en inflación moderada la probabilidad de 
mantenerse en este rango disminuye considerablemente, hasta que las probabilidades de pasar a inflación alta, baja y moderada se igualan.

Los resultados anteriores destacan la importancia de tomar en cuenta la procedencia de cada episodio inflacionario. Las probabilidades de transición a cualquiera de los tres rangos tienen valores y comportamientos muy distintos para los dos tipos de episodio. Además, se puede afirmar que para aquellos países que llegaron al rango de inflación moderada provenientes de niveles de inflación altos, al transcurrir el tiempo, la probabilidad de regresar al nivel alto aumenta, mientras que la probabilidad de mantenerse en el rango de inflación moderada disminuye considerablemente.

Finalmente, se analiza la dependencia de estos resultados del régimen cambiario vigente durante los episodios estudiados. Para ello se tomó una base de datos distinta debido a que se necesita determinar el régimen cambiario vigente durante cada episodio. La nueva muestra comprende 85 países, entre 1970 a 1994. Se estudia en que medida el resultado de un episodio de inflación moderada está relacionado con el régimen cambiario, clasificado como fijo o no fijo ${ }^{3}$. A continuación se presentan las probabilidades de transición para el año siguiente bajo los diferentes regímenes cambiarios.

\section{Gráfica 11}

Probabilidad de transición para episodios de inflación moderada con tipo de cambio fijo (resultados un año después)

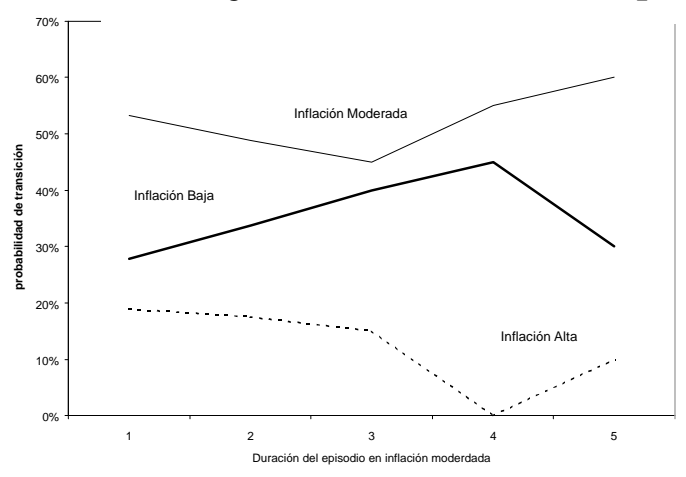

\section{Gráfica 12}

Probabilidad de transición para episodios de inflación moderada con tipo de cambio no fijo (resultados un año después)

\footnotetext{
${ }^{3}$ La clasificación de tipo de cambio fijo incluye aquellas monedas con valor fijo respecto al dólar, la libra esterlina, o los Derechos Especiales de Giro. La clasificación para tipo de cambio no fijo incluye aquellas monedas en flotación, o en régimen flexible, así como aquellas ajustables respecto a una canasta. Los datos provienen del Fondo Monetario Internacional.
} 
A partir de las gráficas 11 y 12 se observa que aquellos países que mantienen un régimen de tipo de cambio fijo, transitan con mayor probabilidad a inflación baja que a inflación alta, mientras que bajo un régimen de tipo de cambio flexible o de flotación estas probabilidades son iguales. A continuación se presentan los resultados para el promedio de los próximos 5 años.

Gráfica 13

Probabilidad de transición para episodios de inflación moderada con tipo de cambio fijo (promedio de cinco años)

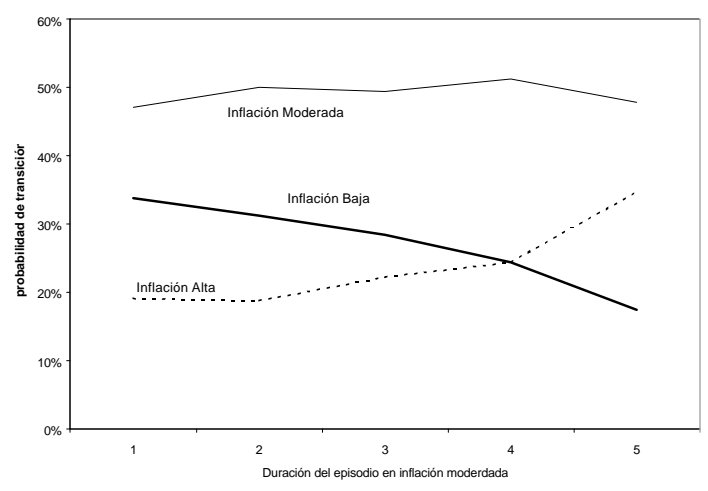

Gráfica 14

Probabilidad de transición para episodios de inflación moderada con tipo de cambio no fijo (promedio de cinco años)

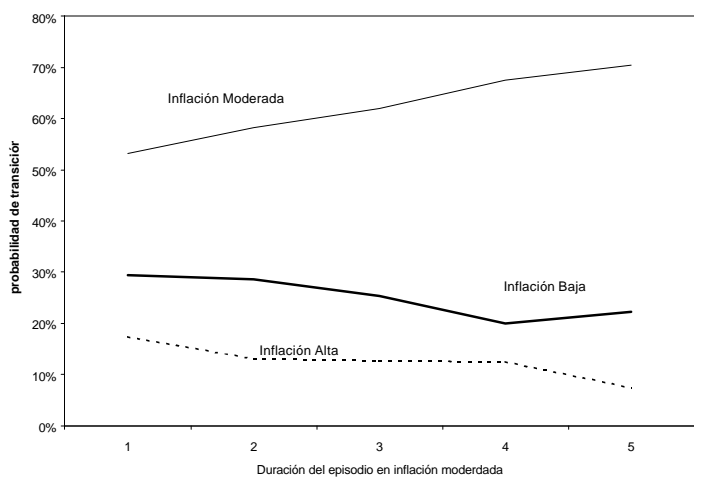

Las gráficas construidas para el promedio de cinco años presentan resultados distintos. En el caso del tipo de cambio fijo, la probabilidad de pasar a inflación alta es importante y aumenta si un país permanece varios periodos en el rango moderado de inflación, a la vez que la probabilidad de llegar a inflación baja desciende. Para el caso del tipo de cambio no fijo estas probabilidades son relativamente constantes. Por otra parte vemos que la probabilidad de que la economía transite a una inflación baja para el promedio de los próximos cinco años es mayor en el caso del régimen de tipo de cambio no fijo, mientras que la probabilidad de transición a inflaciones altas es mayor par el caso de tipo de cambio fijo. Finalmente, la probabilidad de que la inflación permanezca en el rango moderado es mayor para tipos de cambio no fijo. La comparación entre los resultados el año siguiente con aquellos para el promedio de los cinco años permite inferir que en general los programas de estabilización asociados a un tipo de cambio fijo tienen éxito en el corto plazo, sin embargo esta reducción en la tasa de inflación no resulta sostenible. Lo contrario ocurre en el régimen de flotación 0 flexible, donde si bien es menos probable transitar a inflación baja el año siguiente, 
esta probabilidad aumenta de manera importante si se considera el promedio de los cinco años siguientes. En este sentido los procesos desinflacionarios que no dependen de un tipo de cambio fijo han sido más duraderos.

A continuación se resumen los resultados más importantes de esta sección:

(i) La inflación moderada es sustancialmente más inestable que la inflación baja.

(ii) Al aumentar la inflación, ésta se vuelve progresivamente más inestable.

(iii) Cuantos más años un país permanece en inflación moderada, la probabilidad de que ésta se mantenga en este rango aumenta. Sin embargo, esto ocurre a costa de que la inflación se reduzca en el futuro, ya que la probabilidad de transición al rango de inflación elevada se mantiene constante en niveles cercanos al 15\%.

(iv) Para aquellas economías con inflación moderada, la probabilidad de transitar a una inflación alta es sustancialmente mayor si antes del episodio de inflación moderada, dicha economía presentaba niveles altos de inflación que si presentaba una inflación baja.

(v) En general, los programas de estabilización asociados a un tipo de cambio fijo tienen éxito en el corto plazo, sin embargo, esta reducción en la inflación no resulta sostenible. Lo contrario ocurre en el régimen de flotación, donde la probabilidad de transitar a una inflación baja en el próximo año es reducida, pero aumenta de manera importante cuando consideramos un horizonte de 5 años. 


\section{Casos Particulares}

Para complementar el análisis estadístico realizado en la sección anterior, resulta interesante estudiar los casos particulares de economías que hayan experimentado inflaciones moderadas. Por lo tanto en el apéndice se presentan todos los episodios de inflación moderada que duraron 20 más años.

Los casos de mayor interés son en gran medida los que estudian Fischer y D ornbusch [1]. Estos son Corea del Sur, Colombia, Chile, México, Indonesia, Irlanda y España. Además de éstos, aparecen como casos de interés por su estancia en el rango de inflación moderada Ecuador, Nueva Zelandia, Sudáfrica, Turquía, Italia, G recia, Israel y Portugal como países que han tenido una tasa de inflación entre 10 y $20 \%$ por 60 más años consecutivos. Para realizar el análisis de los casos particulares resulta útil distinguir algunos grupos de países que presentan características similares.

Las historias inflacionarias de España, Irlanda, Italia y Portugal se pueden entender como fenómenos esencialmente producidos por los "shocks de oferta" asociados a las crisis petroleras de los años 70's. Estos países tienen una larga historia de inflación reducida, y en todos ellos la inflación alcanzó un nivel máximo menor a 30\%. También hay que destacar que un marco institucional sólido, así como el apoyo y la exigencia de los acuerdos cambiarios europeos fueron elementos importantes que ayudaron a restablecer tasas de inflación bajas. España solicitó pertenecer a la Comunidad Europea en 1977 y se convirtió en miembro en 1985, Irlanda solicitó su entrada en 1967 y se convirtió en miembro en 1973, Italia ha sido miembro de la Comunidad Europea desde sus inicios. Todos estos países han cumplido con los llamados "criterios de convergencia" y están incluidos en la Unión Monetaria Europea. En alguna medida, estos países son interesantes en cuanto a los efectos inflacionarios de los choques de oferta y al rol estabilizador del ancla cambiaria e institucional que proporcionó el Sistema Monetario Europeo y posteriormente las expectativas de la formación de la Unión Monetaria Europea. Aún contando con estos elementos, la reducción de la tasa de inflación a partir de los picos alcanzados en 1984 en Portugal, 1980 en Italia, 1981 en Irlanda y 1976 en España a niveles de un dígito tomó 8, 8, 3 y 5 años respectivamente. 
Gráfica 15

Italia

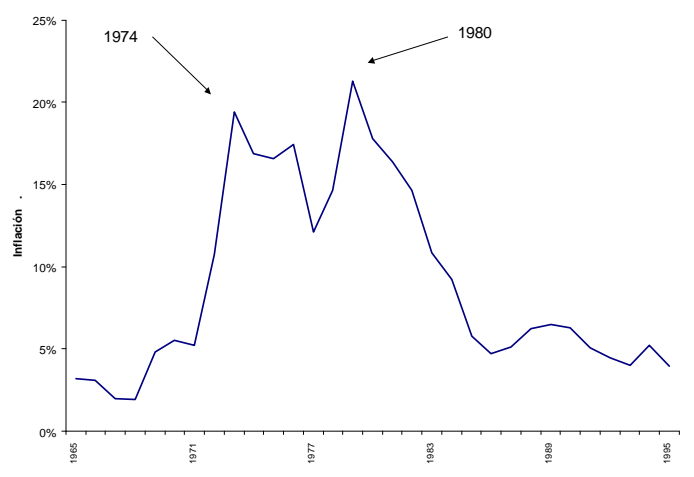

Gráfica 17

Irlanda

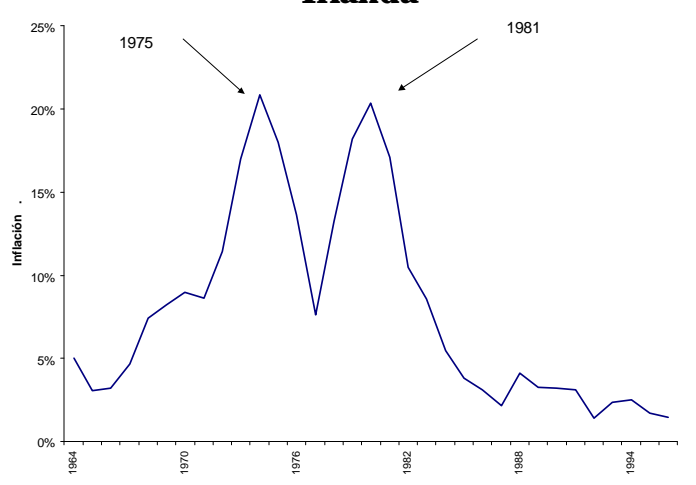

Gráfica 16

España

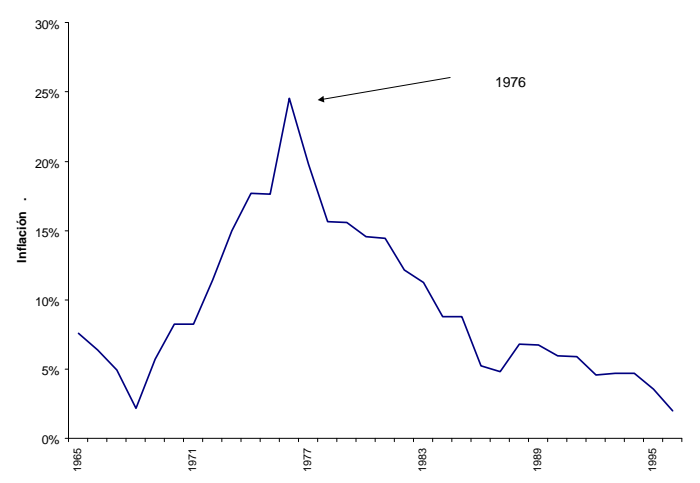

Gráfica 18

Portuaal

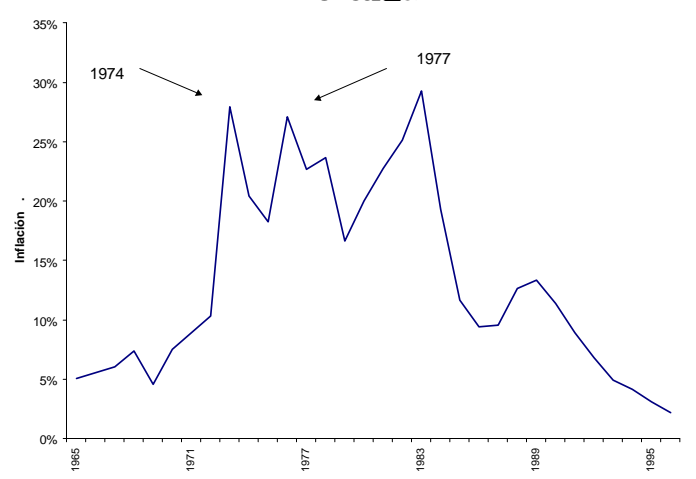

Otro país que podría estar en este conjunto por su historia inflacionaria es Corea. Este país, a pesar de haber tenido una tasa de inflación moderada por seis años consecutivos, y de enfrentar la crisis petrolera de 1982 a la mitad de un plan antiinflacionario, después de esta experiencia ha mantenido tasas de inflación bajas, aún ante las grandes depreciaciones del tipo de cambio experimentadas durante la crisis actual. Aunque durante el periodo de estabilidad se siguieron políticas de tipo de cambio predeterminado, no podemos clasificarlo junto con Portugal, Italia, España e Irlanda básicamente porque no está integrado a ningún sistema cambiario al estilo de los países europeos. 


\section{Gráfica 19}

\section{Corea}

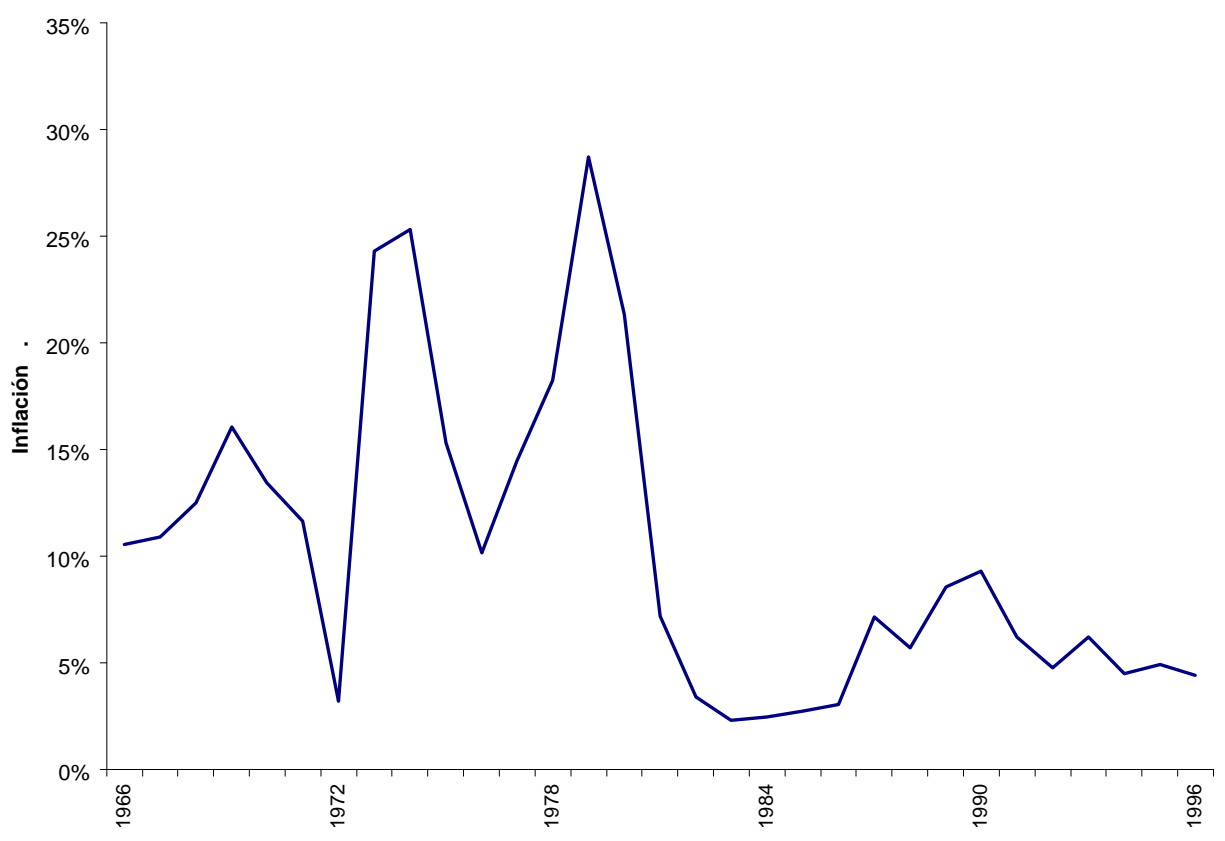

Los casos de Grecia y Nueva Zelandia presentan episodios adicionales de tránsito entre inflación moderada e inflación baja. En Grecia, después de la primera crisis petrolera, se logró estabilizar completamente la inflación en niveles de alrededor de $13 \%$ durante cuatro años consecutivos. Sin embargo, la segunda crisis petrolera elevó la inflación a $25 \%$ dos años después. Este es otro claro ejemplo de la inercia inflacionaria que se genera en los niveles moderados, pues este país no volvió a tener inflación de un dígito hasta 1995. G recia no cumplió con los criterios de convergencia para pertenecer a la Unión Monetaria Europea. En Nueva Zelandia, aún cuando la inflación apareció a partir de los años setentas, ésta no desapareció hasta 1991. Aún con la implementación de un importante programa en 1982 y 83 que la redujo durante dos años a niveles de un dígito, los siguientes tres años la inflación se ubicó alrededor del 15\%. En 1989, Nueva Zelandia se decidió a terminar con la inflación, para lo cual implementó una reforma completa al sistema financiero y a la banca central, haciendo a este instituto completamente independiente del gobierno y con un solo propósito, alcanzar las metas inflacionarias pactadas con el gobierno. 
Gráfica 20

Grecia

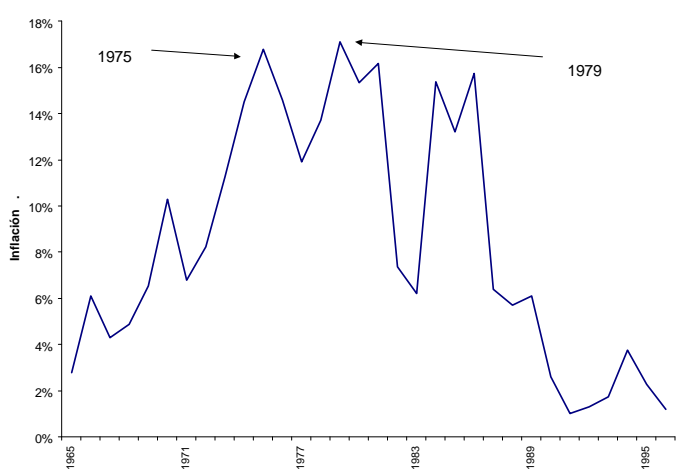

Gráfica 21

N ueva Zelandia

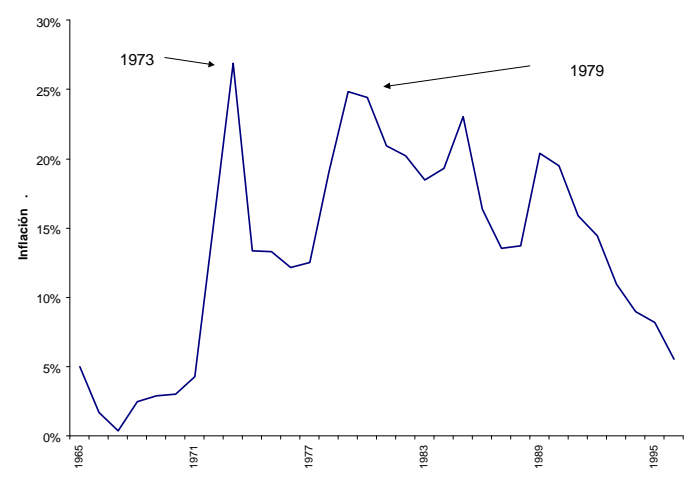

En segundo lugar hay un pequeño grupo de países que después de tener inflación moderada y luego alta por varios periodos, lograron reducir de manera definitiva el nivel de esta tasa. Los ejemplos clásicos son Israel y Chile. Israel mantuvo inflación moderada relativamente estable por un tiempo, pero eventualmente se presentó un circulo vicioso de inflación creciente y devaluaciones progresivas en busca de competitividad. Entonces, al enfrentarse a las crisis petroleras de los setentas y ochentas, la inflación se disparó hasta superar el 300\% en 1985. Después de estos episodios el consenso en torno a la lucha contra la inflación logrado por una amplia coalición de partidos, permitió que se fijara el tipo de cambio al dólar y se iniciara un ajuste fiscal drástico, que permitió reducir la inflación definitivamente. A partir de 1988 se modifico el esquema cambiario, y desde entonces ha sido un esquema de bandas de flotación.
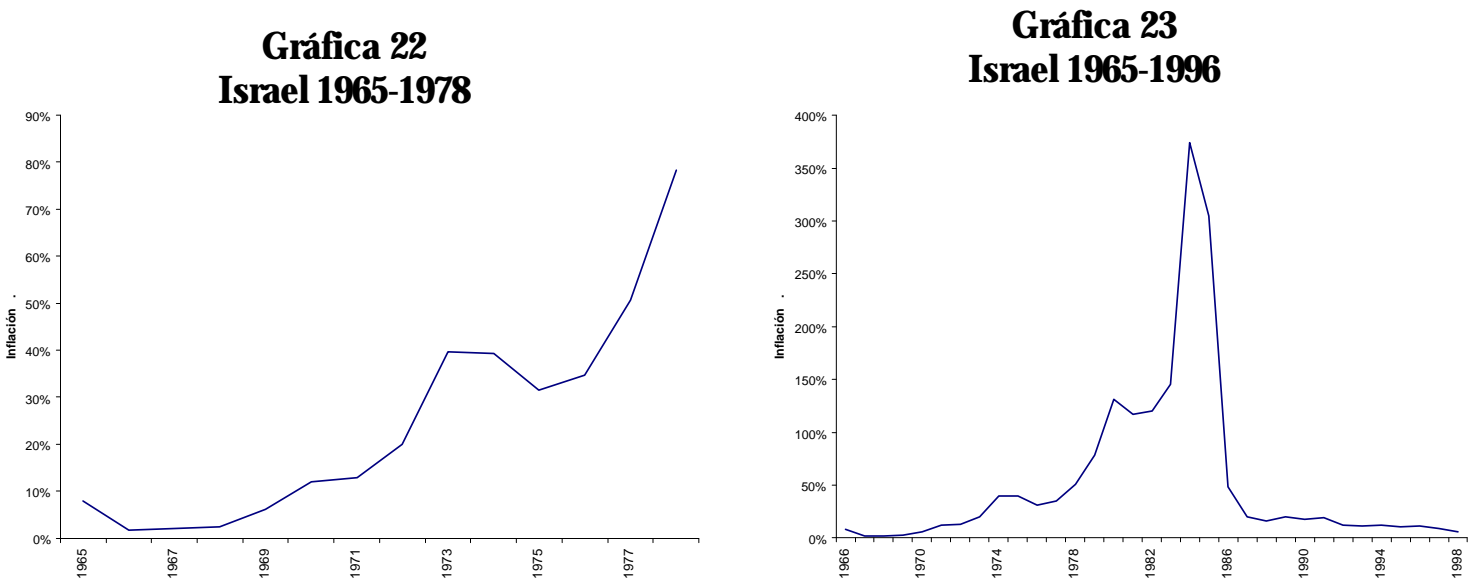

Chile entre 1949 y 1971 tuvo una tasa de inflación muy inestable, y aunque en gran medida los sucesos económicos del final de esta época son atribuibles al gobierno de Allende y al golpe militar posterior, vale la pena destacar que después de haber estado 
siete años (1965-71) con una inflación entre 20 y 40\%, un año después la inflación llegaba al 78\% y dos años después, ésta era de $3600 \%$. El gobierno de Allende, al subir los salarios, incrementar el crédito interno y el déficit público (el déficit era de $25 \%$ del PIB en 1973) provocó un boom económico en 1971 que derivó en una crisis inflacionaria aguda en 1973. Las profundas reformas económicas implementadas por Chile desde los 70's, lograron que el efecto de la crisis de deuda latinoamericana de principios de los 80's no llevara a esta economía a niveles inflacionarios como los de México (100\%), Bolivia (270\%), Argentina (340\%) o Ecuador (48\%), aunque sí hubo un nivel de desempleo exorbitante. El otro episodio chileno de interés se encuentra entre 1986 y 1990. Después de un largo proceso anti-inflacionario, en el cuál la inflación se redujo y se mantuvo en un rango moderado entre 1986 y 1989, en este último año se rompe la tendencia decreciente cuando la inflación rebasa el 20\%. En alguna medida esto fue resultado de la transición a la democracia por la que pasó este país. Aún así, este es un claro ejemplo de la facilidad con la que puede desaparecer la tendencia a la baja en la tasa de inflación. En la década de los 90's la inflación chilena ha restablecido su tendencia a la baja y actualmente se encuentra alrededor de $4.5 \%$. Esto se ha logrado a partir de la autonomía que se le otorgó al banco central en 1990 y a la aplicación de una política monetaria orientada a conseguir el abatimiento de la tasa de inflación a la vez que se trató de evitar el desarrollo de desequilibrios externos importantes, alcanzándose de esta manera una reducción sostenible de la inflación.

Gráfica 24

Chile 1949-1971

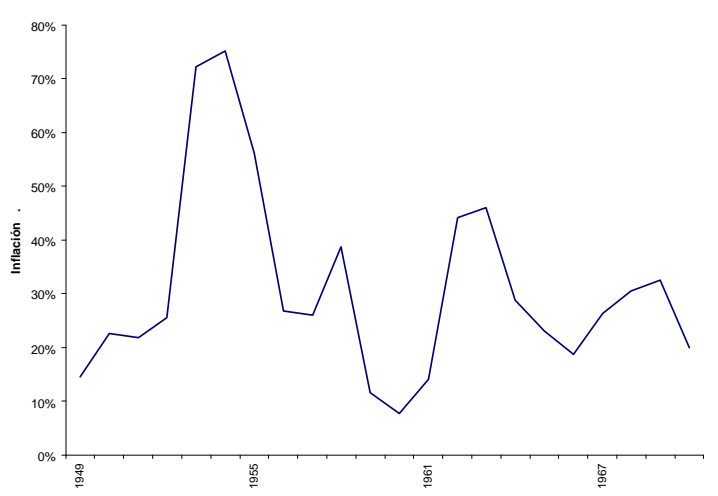

Gráfica 25 Chile 1977-1997



Finalmente podemos agrupar a Colombia, Ecuador y Turquía como los países que han sobrellevado por muy largo tiempo una inflación superior al 10\%, sin caer en la hiperinflación. El caso de Colombia es más interesante, no sólo por su cercanía, sino 
también por su "estabilidad" inflacionaria, pues hace más de 20 años que tiene una inflación entre 15 y $35 \%$. El caso de Turquía es también un caso extremo, ya que estuvo por más de diez años con inflaciones superiores al 50\%, y ésta aumentaba prácticamente año con año con pequeños intervalos en los que se estabiliza, pero sin transitar hacia la hiperinflación.

\section{Gráfica 26}

Turquía

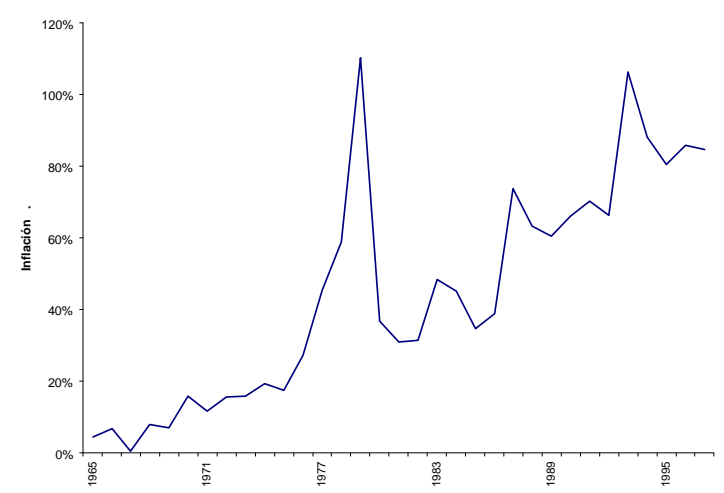

Gráfica 27

Colombia

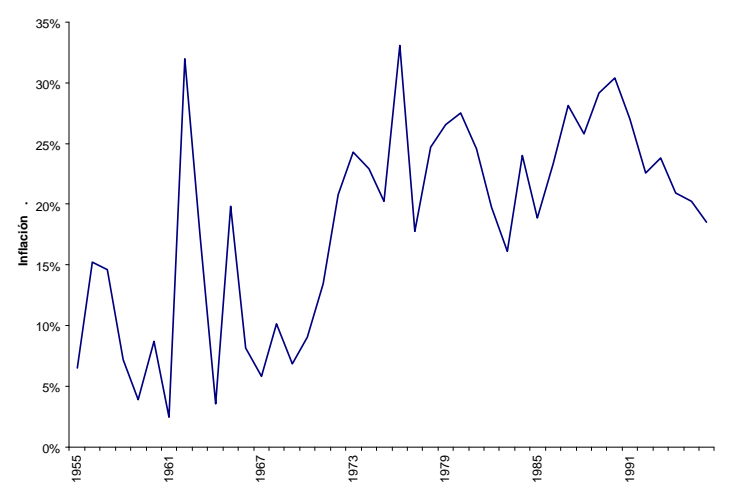

También vale la pena destacar la experiencia con inflación moderada que ha tenido Ecuador. Ecuador mantuvo entre 1975 y 1982 un rango inflacionario moderado y muy estable, sin embargo, al terminar este periodo su inflación aumentó de 16\% en 1982 a 48\% en 1983. El país, sumergido en una crisis de deuda externa, sufrió un choque desfavorable en los términos de intercambio por la reducción en el precio y las reservas de petróleo, por lo que se dio inició a un proceso inflacionario. Aún siguiendo los lineamientos de un programa desinflacionario del FMI por algunos años, Ecuador no ha regresado al rango de inflación moderada, aunque logró reducirla transitoriamente durante 1984 y 1987, ésta volvió a dispararse hasta niveles de 75\% en 1989, sólo dos años después. En la actualidad esta economía se encuentra nuevamente sumergida en una crisis fiscal y bancaria que ha generado un repunte importante en la tasa de inflación. 


\section{Gráfica 28}

Ecuador

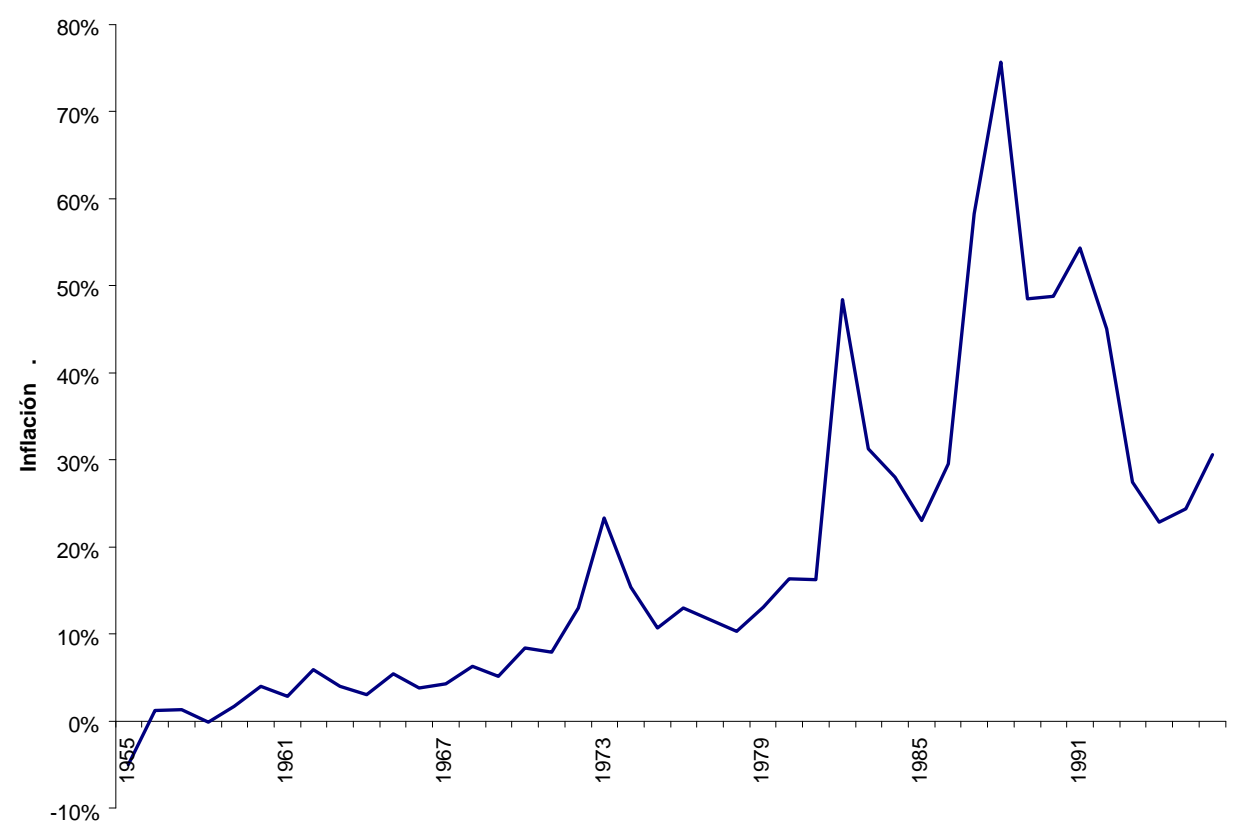

\section{Conclusiones}

A partir de los resultados estadísticos, podemos concluir que la inflación moderada no es un estado permanente, sino que, ya sea por un aumento en la inflación o por una disminución en ésta, la mayoría de los países han salido de este rango inflacionario rápidamente. Aún más importante, encontramos que si bien al pasar un mayor lapso de tiempo con inflación moderada, ésta se vuelve más estable, este fenómeno ocurre a costa de que disminuya la probabilidad de que la inflación descienda. Por lo tanto, los mecanismos que se desarrollan durante estos episodios de inflación moderada, dificultan la disminución de la inflación en el futuro sin afectar las probabilidades de que la inflación aumente. Se detectan también diferencias claras entre aquellos países con inflación moderada que provienen de inflaciones altas y aquellos que provienen de inflaciones bajas ya que los primeros presentan una probabilidad muy superior de transición hacia inflaciones altas que los segundos. Finalmente, la clasificación por 
régimen de tipo de cambio indica que los programas de estabilización basados en el tipo de cambio fijo son exitosos en el corto plazo, pero que esta reducción en la inflación en la mayoría de los casos resultó insostenible. A simismo, se observa que con un régimen de tipo de cambio no fijo, los procesos desinflacionarios han sido más permanentes.

Del estudio de los casos particulares se destaca el hecho que con frecuencia una trayectoria de reducción de la tasa inflacionaria que se estanca en el rango moderado por algunos años pone a una economía en una posición muy vulnerable para enfrentar choques inflacionarios futuros, por lo que en muchos casos se observa nuevamente un incremento de la tasa de aumento de precios (Chile 1961 y 1965, Colombia 1973, Ecuador 1976 y 1985, Grecia 1976, Hungría 1982, Nueva Zelandia 1984 y Turquía 1981). Observamos que sólo Colombia ha mantenido un periodo muy largo de inflación moderada-alta, aparentemente sin muchos problemas (26 años con inflación entre $10 \%$ y $40 \%$ ). A simismo, hay que notar que aunque en varios de estos casos se mantuvo una economía aparentemente saludable con inflación moderada por un periodo prolongado de tiempo, eventualmente, al enfrentarse con perturbaciones negativas, éstas economías transitaron hacia niveles elevados de inflación que dieron lugar a un ajuste anti-inflacionario fuerte con costos importantes para el crecimiento y el desarrollo económico. Estos resultados confirman la importancia de lograr un rápido abatimiento de la inflación a niveles bajos 


\section{Apéndice A}

\section{Episodios de inflación moderada de dos o más años de duración:}

\begin{tabular}{|c|c|c|c|}
\hline \multirow{2}{*}{$\frac{\text { País }}{\text { Afganistan }}$} & \multicolumn{3}{|c|}{ Episodios } \\
\hline & $1986-1987$; & & \\
\hline Algeria & 1976- 1978 ; & 1984- 1985 ; & \\
\hline Antillas Holandesas & $1978-1980$; & & \\
\hline Argentina & $1954-1955$; & & \\
\hline Australia & 1950- 1951 ; & 1973- 1976 ; & $1981-1982$ \\
\hline Bahrain & 1976- 1977 ; & & \\
\hline Bangladesh & 1978- 1981; & 1983- 1985 ; & \\
\hline Barbados & $1971-1972$; & 1978- 1981 ; & \\
\hline Bélgica & 1973- 1974 ; & & \\
\hline Bhutan & $1990-1992$; & & \\
\hline Bolivia & 1977- 1978 ; & $1986-1989$; & $1994-1995$ \\
\hline Botswana & 1975- 1976 ; & 1978- 1982 ; & $1988-1995$ \\
\hline Bukina Faso & 1978- 1979 ; & & \\
\hline Burundi & $1973-1974$; & $1993-1994$; & \\
\hline Camerún & 1972- 1974 ; & $1976-1977$; & $1980-1983$ \\
\hline Canada & 1973- 1974 ; & 1979- 1981 ; & \\
\hline Chile & $1985-1988$; & $1991-1993$; & \\
\hline Chipre & 1979- 1980 ; & & \\
\hline Colombia & $1956-1957$; & $1982-1983$; & \\
\hline Congo. Rep. Del & 1976- 1977 ; & 1980- 1981 ; & \\
\hline Corea & 1966- 1971 ; & $1975-1978$; & \\
\hline Costa de Marfil & 1972- 1975 ; & 1977- 1979 ; & \\
\hline Costa Rica & $1983-1986$; & 1988- 1989 ; & $1995-1996$ \\
\hline Dinamarca & $1976-1977$; & 1979- 1981 ; & \\
\hline Dominica & 1974- 1975 ; & & \\
\hline Ecuador & 1974- 1981 ; & & \\
\hline \multirow[t]{2}{*}{ Egipto } & $1975-1977$; & $1980-1984$; & $1986-1987$ \\
\hline & 1989- 1992 ; & & \\
\hline \multirow{2}{*}{ El Salvador } & 1949- 1950 ; & $1973-1974$; & $1976-1983$ \\
\hline & $1987-1988$; & $1990-1994$; & \\
\hline España & 1956- 1957 ; & 1972- 1975 ; & $1977-1983$ \\
\hline Estados Unidos & 1947- 1948 ; & 1980- 1981 ; & \\
\hline Etiopía & $1976-1978$; & & \\
\hline Fiji & 1972- 1975 ; & 1979- 1980 ; & \\
\hline \multirow[t]{2}{*}{ Finlandia } & 1949- 1950 ; & $1955-1956$; & $1972-1976$ \\
\hline & 1979- 1980 ; & & \\
\hline Francia & 1950- 1951 ; & $1973-1974$; & $1978-1981$ \\
\hline Gabón & $1976-1977$; & $1981-1982$ & \\
\hline Gambia & $1975-1976$; & $1981-1982$; & \\
\hline Ghana & $1971-1973$ & $1990-1991$; & \\
\hline \multirow[t]{2}{*}{ Grecia } & $1974-1978$; & $1983-1984$; & $1986-1988$ \\
\hline & $1990-1993$; & & \\
\hline \multirow[t]{2}{*}{ Guatemala } & $1972-1976$; & 1978- 1980 ; & $1986-1988$ \\
\hline & $1991-1993$; & & \\
\hline Guyana & 1977- 1979 ; & & \\
\hline Haiti & $1973-1974$; & 1978- 1980 ; & $1990-1991$ \\
\hline Honduras & 1978- 1979 ; & & \\
\hline Hungría & 1987- 1988 ; & & \\
\hline \multirow[t]{2}{*}{ India } & $1965-1966$; & 1979- 1980 ; & $1990-1991$ \\
\hline & $1993-1994$; & & \\
\hline \multirow[t]{2}{*}{ Indonesia } & 1968- 1969 ; & $1974-1976$ & $1978-1980$ \\
\hline & $1982-1983$; & & \\
\hline Iran & $1973-1975$; & 1977- 1978 ; & $1981-1983$ \\
\hline \multirow[t]{2}{*}{ Irlanda } & $1972-1973$; & $1975-1976$; & $1978-1979$ \\
\hline & $1981-1982$; & & \\
\hline Islandia & $1961-1963$; & & \\
\hline \multirow[t]{2}{*}{ Islas Solomon } & $1973-1974$; & 1979- 1981 ; & $1985-1988$ \\
\hline & 1990- 1991 ; & & \\
\hline
\end{tabular}

\begin{tabular}{|c|c|c|c|}
\hline País & \multicolumn{3}{|c|}{ Episodios } \\
\hline Israel & $1970-1971$; & 1986- 1987 ; & $1989-1995$ \\
\hline Italia & $1972-1978$; & $1980-1983$; & \\
\hline Jordania & $1972-1976$; & 1978- 1979 ; & \\
\hline Kenia & 1973- 1977 ; & $1979-1980$; & $1982-1984$; \\
\hline & $1987-1990$; & & \\
\hline Las Bahamas & 1973- 1974 ; & 1979- 1980 ; & \\
\hline Lesoto & - 1992 ; & & \\
\hline Madagascar & 1978- 1979 ; & $1984-1986$; & $1991-1992$; \\
\hline Malasia & $1972-1973$; & & \\
\hline Malawi & $1984-1985$; & $1988-1990$; & \\
\hline Maldivas & $1990-1991$; & & \\
\hline Malta & 1979- 1980 ; & & \\
\hline Marruecos & $1950-1951$; & $1980-1981$; & \\
\hline Mauricio & 1974- 1975 ; & $1980-1981$ & 1988- 1989 ; \\
\hline Mexico & $1950-1951$; & $1974-1975$; & $1977-1978$; \\
\hline Namibia & - 1982 & $1984-1991$; & $1993-1994$; \\
\hline Nepal & $1972-1973$; & 1979- 1982 ; & $1985-1986$; \\
\hline & $1990-1991$; & & \\
\hline Nicaragua & $1994-1995$ & & \\
\hline Nigeria & $1968-1970$; & & \\
\hline Noruega & 1979- 1981 ; & & \\
\hline Nueva Zelandia & 1973- 1981 ; & $1984-1986$; & \\
\hline Pakistan & 1979- 1980 ; & $1993-1996$; & \\
\hline Papúa nueva Guinea & $1994-1995$; & & \\
\hline Paraguay & $1991-1992$; & & \\
\hline Peru & $1948-1950$; & $1994-1995$; & \\
\hline Philipinas & $1978-1982$; & $1988-1990$; & \\
\hline Polonia & $1983-1985$; & & \\
\hline Portugal & $1984-1985$; & $1988-1990$; & \\
\hline Reino Unido & $1975-1976$ & $1978-1980$; & \\
\hline República Central Africana & $1981-1982$ & & \\
\hline República Dominicana & 1972- 1974 ; & & \\
\hline Ruanda & $1976-1978$; & & \\
\hline S.Vicente y Granadinas & $1975-1976$; & $1978-1980$; & \\
\hline Samoa & $1981-1983$ & & \\
\hline Senegal & $1972-1973$; & $1981-1984$; & \\
\hline Seycheles & $1974-1980$; & & \\
\hline Sierra leone & $1973-1975$ & & \\
\hline Siria & $1973-1976$; & 1979- 1981 ; & 1988- 1989 ; \\
\hline & $1991-1993$; & & \\
\hline Sri Lanka & $1977-1978$ & $1980-1983$; & $1987-1988$; \\
\hline & $1990-1992 ;$ & & \\
\hline Sta. Lucía & $1979-1980$; & & \\
\hline Sud-Africa & 1973- 1991 ; & & \\
\hline Sudán & $1971-1972$ & $1976-1977$; & \\
\hline Suecia & $1975-1976$; & 1979- 1980 ; & \\
\hline Surinam & 1978- 1979 ; & $1984-1985$; & \\
\hline Swazilandia & $1972-1974$; & $1978-1979$; & $1981-1983$; \\
\hline & $1985-1987$; & $1989-1990$; & $1992-1995$; \\
\hline Tailandia & 1979- 1980 ; & & \\
\hline Tanzania & $1966-1968$; & $1972-1973$; & $1995-1996$ \\
\hline Tirnidad y Tobago & $1974-1983$; & $1988-1989$; & \\
\hline Togo & $1973-1975$; & 1979- 1981 ; & \\
\hline Turkía & $1970-1975$; & & \\
\hline Uruguay & $1950-1951$; & $1956-1957$; & \\
\hline Venezuela & $1983-1985$; & & \\
\hline Zimbawe & $1974-1976$ & $1980-1981$ & $1985-1986$ \\
\hline & $1988-1989$; & & ; \\
\hline
\end{tabular}




\section{Bibliografía}

[1] Barro, R. J. (1995). "Inflation and economic growth. Bank of England Quarterly Bulletin, 35, May, 166-176.

[2] Barro, R. J. (1997). "Determinants of economic growth. MIT Press, Cambridge, Mass.

[3] Bosworth, Barry P. ed. (1994), "The Chilean economy: policy lessons and challenges". Ed. by Barry P. Bosworth, Rudiger Dombusch and Raul Laban. -Washington Brookings Institute.

[4] Bruno, M. and Easterly, W. (1996). "Inflation and growth: in search of a stable relationship”. Federal Reserve Bank of St. Louis Review, 78(3), 139-146.

[5] De G regorio, J. (1992). "The effects of inflation on economic growth". European Economic Review, 36, 417-24.

[6] De G regorio, J. (1993). "Inflation, taxation and long-run growth". Journal of Monetary Economics, 31, 271-98.

[7] Dombusch, Rudiger y Stanley Fischer (1991), "Moderate inflation", Policy Research Working Paper 807, World Bank, Washington D.C.

[8] Fischer, S. (1993). "The role of macroeconomic factors in growth". Journal of Monetary Economics, 32(3), 485-511.

[9] G hosh, Atish y Phillips, Steven (1998), "Warning: Inflation may be harmful to your growth", IMF Staff Papers 45(4), 672-711.

[10] Leiderman, Leonardo (1993), "Inflation and disinflation: the Israeli experiment". Chicago University of Chicago Press.

[11] Sarel, M. (1996). "Nonlinear effects of inflation on economic growth". IMF Staff Papers, 43(1), 199-215 